PROCEEDINGS OF THE

AMERICAN MATHEMATICAL SOCIETY

Volume 129, Number 10, Pages 3049-3056

S 0002-9939(01)05932-9

Article electronically published on April 9, 2001

\title{
ON BELINSKII CONFORMALITY IN COUNTABLE SETS OF POINTS
}

\author{
VLADIMIR I. RYAZANOV AND MATTI K. VUORINEN \\ (Communicated by Albert Baernstein II) \\ Dedicated to Professor P.P. Belinskii
}

\begin{abstract}
The local behavior of plane quasiconformal mappings is investigated. In particular, generalizing the well-known Reich-Walczak problem, we study the possibility for a quasiconformal mapping to be conformal in the sense of Belinskii at a prescribed point or in a prescribed set of points when the modulus of the complex dilatation is a fixed measurable function. The notion of the Belinskii conformality is related to the conception of asymptotical rotations by Brakalova and Jenkins.
\end{abstract}

\section{INTRODUCTION}

By one of the analytic definitions of quasiconformality (cf., e.g., [LV], p. 176, $\mathrm{A}$, pp. 24,33), a sense-preserving homeomorphism of the complex plane $f: \mathbb{C} \rightarrow \mathbb{C}$, of the class $W_{1, l o c}$, is called $Q$-quasiconformal, $Q \in[1, \infty)$, if it satisfies the Beltrami equation

$$
f_{\bar{z}}=\mu(z) f_{z} \quad \text { a.e. }
$$

where, as usual, $f_{\bar{z}}=\left(f_{x}+i f_{y}\right) / 2, f_{z}=\left(f_{x}-i f_{y}\right) / 2, z=x+i y$, and $\mu: \mathbb{C} \rightarrow \mathbb{C}$ is a measurable function such that

$$
p(z)=\frac{1+|\mu(z)|}{1-|\mu(z)|} \leq Q \quad \text { a.e. }
$$

The quantities $\mu(z)$ and $p(z)$ are called the complex dilatation and the dilatation of the mapping $f$ at the point $z$, respectively. Conversely, given $Q \in[1, \infty)$ and a measurable function $\mu$ of a subdomain $D$ of the plane satisfying the inequality (1.2), there exists a $Q$-quasiconformal homeomorphism of $D$ whose complex dilatation is equal to $\mu$ a.e. ([LV], p. 204).

In the study of pointwise differentiability of quasiconformal mappings, a series of fundamental results has been obtained. O. Teichmüller, H. Wittich, P.P. Belinskii and $\mathrm{O}$. Lehto have established that, for a quasiconformal mapping $f$, the integral

Received by the editors October 26, 1999 and, in revised form, March 9, 2000.

1991 Mathematics Subject Classification. Primary 30C62; Secondary 30 G15.

Key words and phrases. Quasiconformal mappings, local behavior, conformality, asymptotical rotations. 
condition

$$
\int_{\left|z-z_{0}\right|<\delta} \frac{|\mu(z)|}{\left|z-z_{0}\right|^{2}} d x d y<\infty,
$$

for some $\delta>0$, implies the conformality of $f$ at the point $z_{0}$; see, e.g., $[\mathrm{T}],[\mathrm{W}]$, [B1], [B2], [L] and [LV].

In [RW], it was conjectured that, for whatever the fixed dilatation $p(z)$, it is possible to choose the $\operatorname{argument} \arg \mu(z)$ of the complex dilatation in such a way that the corresponding quasiconformal mapping be conformal at an arbitrary prescribed point $z_{0} \in \mathbb{C}$. In other words, if $K(z): \mathbb{C} \rightarrow[1, Q]$ is an arbitrary measurable function, then there is a quasiconformal mapping $f: \mathbb{C} \rightarrow \mathbb{C}$ with the local dilatation

$$
p(z)=K(z) \quad \text { a.e. in } \mathbb{C}
$$

such that $f$ is conformal at the point $z_{0}$.

In [RW], a particular solution of the problem has also been given. More precisely, E. Reich and $\mathrm{H}$. Walczak have shown that, for every measurable function $\varphi(r)$ : $(0,1) \rightarrow[1, Q]$, there is a quasiconformal mapping $f$ of the unit disk $\mathbb{D}=\{z \in \mathbb{C}$ : $|z|<1\}$ onto itself that has the dilatation

$$
p(z)=\varphi(|z|) \quad \text { a.e. in } \mathbb{D}
$$

and that is conformal at the origin; see also [LV], pp. 248-249.

We consider similar problems with respect to the so-called conformality in the sense of Belinskii at a single point as well as in a prescribed set of points. A related notion of the so-called asymptotic rotation is studied by J.A. Jenkins and M. Brakalova [BJ1], [BJ2]. Both notions inherit the main geometrical properties of the usual conformality.

\section{Preliminary COMments And the Main RESUlt}

Recall that a mapping $f$ is called conformal at a point $z_{0}$ if there is a nonzero derivative of $f$ in the sense of the complex analysis, $f^{\prime}\left(z_{0}\right) \neq 0$. In other words, $f$ has a total differential at $z_{0}$,

$$
\Delta f=f^{\prime}\left(z_{0}\right) \Delta z+o(|\Delta z|)
$$

with a nonsingular linear part. The main geometric properties of a mapping $f$ which is conformal at a point are the preservation of angles, infinitesimal disks and the moduli of infinitesimal rings centered at the point.

As the following example of Shabat [Sh] shows,

$$
w=z(1-\log |z|), \quad|z|<\delta<1,
$$

the mapping $w=f(z), f(0)=0$, may be nondifferentiable in the usual sense even if the complex dilatation $\mu(z)$ is continuous with

$$
\mu(z) \rightarrow 0 \quad \text { as } z \rightarrow 0 .
$$

However, if $\mu(z)$ is continuous at a point $z_{0}$, then, as was first observed by Belinskii (see [B1], p. 41), the mapping $w=f(z)$ is differentiable in the sense that

$$
\Delta w=A(\rho)\left(\Delta z+\mu_{0} \Delta \bar{z}+o(\rho)\right)
$$


where $\mu_{0}=\mu\left(z_{0}\right)$ and $A(\rho)$ depends on $\rho=\left|\Delta z+\mu_{0} \Delta \bar{z}\right|$ while $o(\rho) / \rho \rightarrow 0$ as $\rho \rightarrow 0$. Here we must stress that in this case, as opposed to the usual differentiability, $A(\rho)$ may fail to have a limit value as $\rho \rightarrow 0$ but

$$
\lim _{\rho \rightarrow 0} \frac{A(t \rho)}{A(\rho)}=1
$$

for each fixed $t>0$; see [R1, p. 290 .

The differentiability in the sense of (2.4) together with the additional condition (2.5) is called differentiability in the sense of Belinskii. Also, $\mu_{0}$ in the relation (2.4) will not necessarily be equal to $\mu\left(z_{0}\right)$ if $\mu(z)$ is not continuous at the point $z_{0}$. If $\mu_{0}=0$, the mapping $f$ is said to be conformal in the sense of Belinskii at $z_{0}$. Thus, conformality in the sense of Belinskii means that at the point $z_{0}$,

$$
\Delta w=A(\rho)(\Delta z+o(\rho))
$$

where $\rho=|\Delta z|$ and (2.5) holds.

In R1] (see also GR]) a few criteria of conformality in the sense of Belinskii were found. One of these criteria, a necessary and sufficient condition, is the (complex) asymptotic homogeneity of a quasiconformal mapping $f: \mathbb{C} \rightarrow \mathbb{C}, f(0)=0$, at the origin, i.e.,

$$
\lim _{z \rightarrow 0} \frac{f(z \zeta)}{f(z)}=\zeta
$$

for each fixed $\zeta \in \mathbb{C}$. Moreover, the pointwise limit relation (2.7) is equivalent to one which is uniform in each compact subset of $\mathbb{C}$ with respect to the parameter $\zeta$.

Simultaneously, conformality in the sense of Belinskii is equivalent to the (real) asymptotic homogeneity of a quasiconformal mapping $f: \mathbb{C} \rightarrow \mathbb{C}, f(0)=0$, at the origin, i.e.,

$$
\lim _{z \rightarrow 0} \frac{f(z t)}{f(z)}=t
$$

for each fixed $t>0$. Sometimes, we write the relations (2.7) and (2.8) in the form $f(z \zeta) \sim \zeta f(z)$ and $f(z t) \sim t f(z)$ as $z \rightarrow 0$.

From (2.7) we obtain also asymptotic preservation of angles between rays emanating from the origin in the direction of the corresponding points,

$$
\lim _{z \rightarrow 0}\{\arg f(z \zeta)-\arg f(z)\}=\arg \zeta,
$$

and preservation of the moduli of infinitesimal rings,

$$
\lim _{z \rightarrow 0} \frac{|f(z \zeta)|}{|f(z)|}=|\zeta|
$$

for each fixed $\zeta \in \mathbb{C} \backslash\{0\}$.

2.11. Remark. Thus, the main geometric properties of usual conformality are inherited by the Belinskii conformality. These two properties (2.9) and (2.10) are characteristic for conformality in the sense of Belinskii. Note also that the pointwise limit relations (2.9) and (2.10) are equivalent to the uniform limit relations (2.9) and (2.10) with respect to the parameter $\zeta \in \mathbb{C}, c^{-1} \leq|\zeta| \leq c$, and, in particular, in the unit circle $|\zeta|=1$. 
Another more restrictive notion that is close to conformality in the sense of Belinskii was defined by M. Brakalova and J.A. Jenkins BJ1, BJ2. They say that $f(z)$ is asymptotically a rotation on circles as $z \rightarrow 0$, if, first,

$$
|f(z)| \sim A|z| \quad \text { as } z \rightarrow 0, \quad A>0
$$

and, second, for an appropriate choice of the arguments,

$$
\arg f\left(r e^{i \vartheta_{2}}\right)-\arg f\left(r e^{i \vartheta_{1}}\right)-\left(\vartheta_{2}-\vartheta_{1}\right)
$$

tends to zero uniformly in $\vartheta_{1}$ and $\vartheta_{2}$ as $r$ tends to zero. Thus, the main difference of conformality in the sense of Belinskii from the asymptotic rotations is in a more general relation (2.5) instead (2.12) with fixed $A>0$.

The positive solution to the Reich-Walczak problem in the case of a single point with respect to conformality in the sense of Belinskii has been given in [R2].

2.14. Proposition. Let $K(z): \mathbb{C} \rightarrow[1, Q]$ be an arbitrary measurable function. Then there is a quasiconformal mapping $f: \mathbb{C} \rightarrow \mathbb{C}$ with the local dilatation $p(z)=$ $K(z)$ a.e. such that $f$ is conformal in the sense of Belinskii at a prescribed point $z_{0} \in \mathbb{C}$.

The main result of the present paper is the following statement on the simultaneous conformality by Belinskii in countable sets of points.

2.15. Theorem. Let $K(z): \mathbb{C} \rightarrow[1, Q]$ be a measurable function and let $S=$ $\left\{z_{n}\right\}_{n=1}^{\infty}$ be a countable subset of $\mathbb{C}$. Then there is a quasiconformal mapping $f$ : $\mathbb{C} \rightarrow \mathbb{C}$ with the local dilatation $p(z)=K(z)$ a.e. such that $f$ is conformal in the sense of Belinskii at all points $z_{n}, n=1,2, \cdots$.

In particular, we have the following interesting consequences.

2.16. Corollary. Let $K(z): \mathbb{C} \rightarrow[1, Q]$ be an arbitrary measurable function. Then there is a quasiconformal mapping $f: \mathbb{C} \rightarrow \mathbb{C}$ with the local dilatation $p(z)=K(z)$ a.e. such that $f$ is conformal in the sense of Belinskii at all points $z \in \mathbb{C}$ with rational coordinates.

2.17. Corollary. Let $Q>1$ be an arbitrary number. Then there is a quasiconformal mapping $f: \mathbb{C} \rightarrow \mathbb{C}$ with the local dilatation $p(z)=Q=$ constant a.e. such that $f$ is conformal in the sense of Belinskii in an arbitrarily prescribed countable subset of $\mathbb{C}$.

\section{A proof of the MAIN RESUlt}

The proof of Theorem 2.15 is based on Proposition 2.14 and two lemmas. The first of them is the following comparison lemma and the second one, the so-called covering lemma, will be formulated below.

3.1. Lemma. Let $g$ and $f: \mathbb{C} \rightarrow \mathbb{C}, f(0)=g(0)=0$, be Q-quasiconformal mappings with the complex dilatations $\mu$ and $\nu$ such that

$$
\lim _{\varepsilon \rightarrow 0} \frac{1}{\pi \varepsilon^{2}} \int_{|z|<\varepsilon}|\mu(z)-\nu(z)| d x d y=0 .
$$

Then

$$
g(z)=\varphi(f(z)), \quad z \in \mathbb{C},
$$


where the quasiconformal mapping $\varphi: \mathbb{C} \rightarrow \mathbb{C}$ is conformal in the sense of Belinskii at the origin.

3.4. Corollary. Under the conditions of Lemma [3.1, the mapping $f$ is conformal in the sense of Belinskii at the origin if and only if $g$ is.

A point $z_{0}$ in a measurable set $E$ is called a density point of $E$ if

$$
\lim _{\varepsilon \rightarrow 0} \frac{\operatorname{meas} E \cap D\left(z_{0}, \varepsilon\right)}{\operatorname{meas} D\left(z_{0}, \varepsilon\right)}=1
$$

where $D\left(z_{0}, \varepsilon\right)$ is the open disk with center $z_{0}$ and radius $\varepsilon$.

3.5. Corollary. Let $g$ and $f: \mathbb{C} \rightarrow \mathbb{C}, f(0)=g(0)=0$, be Q-quasiconformal mappings with the complex dilatations $\mu$ and $\nu$ such that

$$
\mu(z)=\nu(z), \quad z \in E \subset \mathbb{C},
$$

where $E$ is an arbitrary measurable set for which 0 is a density point. Then the mapping $f$ is conformal in the sense of Belinskii at the origin if and only if $g$ is.

In what follows, $\mathfrak{F}_{Q}$ denotes the class of all the $Q$-quasiconformal self-mappings of the extended complex plane $\overline{\mathbb{C}}=\mathbb{C} \bigcup\{\infty\}$ normalized in the following way:

$$
f(0)=0, \quad f(1)=1, \quad f(\infty)=\infty .
$$

Proof of Lemma 3.1. First of all, the complex dilatation of the mapping $\varphi=g \circ f^{-1}$ is

$$
\kappa(z)=\left\{\frac{\mu-\nu}{1-\mu \bar{\nu}} \cdot \exp \left(2 i \arg f_{w}\right)\right\} \circ f^{-1}(z), \quad z \in \mathbb{C} ;
$$

see, e.g., $[\mathrm{A}]$, p. 9. Thus, the family of the mappings

$$
\varphi(z ; t)=\varphi(t z) / \varphi(t) \in \mathfrak{F}_{Q}, \quad t>0,
$$

corresponds to the family of their complex dilatations

$$
\kappa(z ; t)=\kappa(t z)=\alpha(h(z ; t) ; t), \quad t>0 .
$$

Here

$$
h(z ; t)=f^{-1}(t z) / f^{-1}(t) \in \mathfrak{F}_{Q}, \quad t>0
$$

and

$$
\alpha(w ; t)=\left\{\frac{\mu-\nu}{1-\mu \bar{\nu}} \cdot \exp \left(2 i \arg f_{w}\right)\right\} \circ(w \zeta(t)), \quad w \in \mathbb{C},
$$

where $\zeta(t)=f^{-1}(t) \rightarrow 0$ as $t \rightarrow 0$.

However, making changes of variables $\varepsilon=R|\zeta(t)|$ and $z=w \zeta(t)$ in (3.2), we obtain that

$$
\lim _{t \rightarrow 0} \frac{1}{\pi R^{2}} \int_{|w|<R}|\mu(w \zeta(t))-\nu(w \zeta(t))| d u d v=0
$$

for each fixed $R>0$. Here $w=u+i v$. It follows that

$$
\mu(w \zeta(t))-\nu(w \zeta(t)) \rightarrow 0 \quad \text { as } t \rightarrow 0
$$

in measure in the disk $D(0, R)$, for each $R>0$, i.e., in measure in $\mathbb{C}$. Hence we conclude that

$$
\alpha(w ; t) \rightarrow 0 \quad \text { as } t \rightarrow 0
$$


in measure in $\mathbb{C}$ because

$$
\left|\left\{\frac{1}{1-\mu \bar{\nu}} \cdot \exp \left(2 i \arg f_{w}\right)\right\} \circ(w \zeta(t))\right| \leq \frac{1}{1-q^{2}}
$$

where $q=(Q-1) /(Q+1)$.

Further, since the homeomorphisms $h(z ; t) \in \mathfrak{F}_{Q}, t>0$, are locally absolutely equicontinuous (see G]), also

$$
\kappa(z ; t) \rightarrow 0 \quad \text { as } t \rightarrow 0
$$

in measure in $\mathbb{C}$.

Finally, in view of the sequential compactness of the class $\mathfrak{F}_{Q}$, (see, e.g., [LV], p. 176) and the well-known Bers-Bojarskii convergence theorem (see, e.g., [Be], $[\mathrm{Bo}]$ and [LV], p. 197),

$$
\varphi(z ; t)=\frac{\varphi(t z)}{\varphi(t)} \rightarrow z \quad \text { as } t \rightarrow 0
$$

that is, equivalent to conformality in the sense of Belinskii of the mapping $\varphi$ at the origin; see [R1], p. 290, and [R3], p. 201.

3.7. Lemma. Let $S=\left\{z_{n}\right\}_{n=1}^{\infty}$ be an arbitrary countable subset of $\mathbb{C}$. Then there is a disjoint collection of closed sets $E_{n} \subset \mathbb{C}$ such that $z_{n} \in E_{n}$ is a density point of $E_{n}$ for each $n=1,2, \cdots$.

Proof of Lemma 3.7. First, we prove that there is a collection of closed sets $L_{n}$, generally speaking, with a nonempty intersection such that, for each $n=1,2, \cdots$, the point $z_{n} \in L_{n}$ is a density point for $L_{n}$ and $L_{n} \cap S \backslash\left\{z_{n}\right\}=\emptyset$.

Indeed, denote by $D\left(z_{n}, r_{m}\right)$ the open disk with the center $z_{n}$ and radius $r_{m}=$ $1 / m, m=0,1,2, \cdots$. As is well-known, every countable subset of $\mathbb{C}$ has zero Lebesgue measure and hence by the regularity of the measure (see, e.g., [S]) there exist open sets $\Omega_{n}^{(m)}, m=0,1,2, \cdots$, such that

$$
S \cap D\left(z_{n}, r_{m}\right) \backslash\left\{z_{n}\right\} \subset \Omega_{n}^{(m)} \subset D\left(z_{n}, r_{m}\right) \backslash\left\{z_{n}\right\}
$$

where meas $\Omega_{n}^{(m)} \leq 2^{-m}$ and we may assume that, for each fixed $n=1,2, \cdots$, the sequence of the sets $\Omega_{n}^{(m)}, m=0,1,2, \cdots$, is decreasing.

Set $L_{n}=\mathbb{C} \backslash \Omega_{n}$ where

$$
\Omega_{n}=\bigcup_{m=0}^{\infty} \Omega_{n}^{(m)} \backslash \overline{D\left(z_{n}, r_{m+1}\right)}
$$

We see directly by the construction that $L_{n} \cap S \backslash\left\{z_{n}\right\}=\emptyset, z_{n} \in L_{n}$, and $L_{n}$ is closed for each $n=1,2, \cdots$. It remains to show that $z_{n}$ is a density point for $L_{n}$. To verify this we write

$$
\begin{gathered}
\alpha_{n, \rho}=\frac{\operatorname{meas} D\left(z_{n}, \rho\right) \backslash L_{n}}{\operatorname{meas} D\left(z_{n}, \rho\right)}=\frac{\operatorname{meas} D\left(z_{n}, \rho\right) \cap \Omega_{n}}{\operatorname{meas} D\left(z_{n}, \rho\right)} \\
\leq \frac{\sum_{m=k-2}^{\infty} \operatorname{meas} \Omega_{n}^{(m)}}{\operatorname{meas} D\left(z_{n}, \rho\right)} \leq \frac{k^{2}}{\pi} \sum_{m=k-2}^{\infty} 2^{-m} \leq \frac{k^{2}}{2^{k-3}} \rightarrow 0 \quad \text { as } \quad k \rightarrow \infty
\end{gathered}
$$


where $k=1+\left[\rho^{-1}\right]$ and $\left[\rho^{-1}\right]$ is the integral part of the number $1 / \rho$. Thus, for each fixed $n=1,2, \cdots$, we have the limit relation $\alpha_{n, \rho} \rightarrow 0$ as $\rho \rightarrow 0$. It just means that the point $z_{n}$ is a density point of the set $L_{n}$.

Now we construct by induction mutually disjoint closed sets $E_{n}$ making use of $L_{n}$. More precisely, we let $E_{1}=L_{1} \cap C_{1}$ where $C_{1}$ is a closed disk with center $z_{1}$ and arbitrary radius $\rho_{1}$. If $E_{1}, \cdots, E_{n}$ have already been given we set

$$
E_{n+1}=L_{n+1} \cap C_{n+1}
$$

where $C_{n+1}$ is the closed disk with center $z_{n+1}$ and radius

$$
\rho_{n+1}=\frac{1}{2} \min _{z \in \bigcup_{k=1}^{n} E_{k}}\left|z_{n+1}-z\right| .
$$

Immediately by the construction we see that $E_{n}$ are closed, mutually disjoint and $z_{n} \in E_{n}$ are density points of the corresponding $E_{n}$ for each fixed $n=1,2, \cdots$.

We complete the section by a proof of the main result.

Proof of Theorem 2.15. First, by Proposition 2.14, for each $n=1,2, \cdots$ there is a quasiconformal mapping $f_{n}: \mathbb{C} \rightarrow \mathbb{C}$ with the dilatation $p_{n}(z)=K(z)$ a.e. in $\mathbb{C}$ such that $f_{n}$ is conformal in the sense of Belinskii at the point $z_{n}$. Let $\mu_{n}$ be the complex dilatation of $f_{n}, n=1,2, \cdots$.

Then by the covering lemma there is a disjoint collection of measurable sets $E_{n} \subset \mathbb{C}$ such that $z_{n} \in E_{n}$ is a density point of the corresponding $E_{n}$ for each $n=1,2, \cdots$.

Further, by the existence theorem (see, e.g., $[\mathrm{A}],[\mathrm{Bo}]$ and $[\mathrm{LV}]$ ), there is a $Q^{-}$ quasiconformal mapping $f: \mathbb{C} \rightarrow \mathbb{C}$ with the complex dilatation

$$
\mu(z)=\mu_{n}(z), \quad z \in E_{n},
$$

and, say,

$$
\mu(z)=k(z), \quad z \in \mathbb{C} \backslash \bigcup_{n=1}^{\infty} E_{n},
$$

where $k(z)=(K(z)-1) /(K(z)+1)$.

Finally, by the comparison lemma, $f$ is conformal in the sense of Belinskii at each point $z_{n}, n=1,2, \cdots$.

\section{REFERENCES}

[A] Ahlfors L., Lectures on Quasiconformal Mappings, Princeton, NJ, Van Nostrand, 1966. MR 36:336

[B1] Belinskil P.P., General properties of quasiconformal mappings, Novosibirsk, Nauka, 1974 (Russian). MR 53:11054

[B2] Belinski P.P., Behavior of a quasiconformal mapping at an isolated singular point, Uchenye Zapiski Lvovski Gosudarstvennyi Universitet. Ser. Mekhaniko-Matematichna 29 (1954), 58-70 (Russian). MR 18:740c

[Be] Bers L., On a theorem Mori and the definition of quasiconformality, Trans. Amer. Math. Soc. 84 (1957), 78-84. MR 18:646d

[Bo] Bojarski B. Generalized solutions of PDE system of the first order and elliptic type with discontinuous coefficients, Mat. Sb. 43 (85) (1957), 451-503 (Russian). MR 21:5058

[BJ1] Brakalova M. AND J.A. Jenkins, On the local behavior of certain homeomorphisms, Kodai Math. J. 17 (1994), 201-213. MR 95h:30019

[BJ2] Brakalova M. and J.A. Jenkins, On the local behavior of certain homeomorphisms. II, Notes of Sci. Sem. of POMI 237 (1997), 11-20. MR 2000e:30034 
[G] GeHRING F.W., $L^{p}$-integrability of the partial derivatives of quasiconformal mappings, Acta Math. 130 (1973), 265-277. MR 53:5861

[GR] Gutlyanskit V.Ya. and Ryazanov V.I., On the theory of local behavior of quasiconformal mappings, Izv. AN Rossii, Math. Ser., 59, no. 3 (1995), 31-58 (Russian). MR 96h:30025

[L] LeHto O., On the differentiability of quasiconformal mappings with prescribed complex dilatation, Ann. Acad. Sci. Fenn. Ser. A I 275 (1960), 1-28. MR 23:A3260

[LV] Lehto O., Virtanen K., Quasikonforme Abbildungen, Berlin etc., Springer-Verlag, 1965. MR 32:5872

[RW] Reich E. AND WalczAK H., On the behavior of quasiconformal mappings at a point, Trans. Amer. Math. Soc. 117 (1965), 338-351. MR 31:345

[R1] Ryazanov V.I., Criterion of differentiability by Belinskii and its consequences, Ukrain. Mat. Zh. 44 (1992), 289-294 (Russian); translation in Ukrainian Math. J. 44 (1992), no. 2, 254-258. MR 93g:30027

[R2] Ryazanov V.I., Solution of the Reich-Walczak problem on conformality by BelinskiiLavrent'ev, Ukrain. Mat. Zh. 44 (1992), 1406-1411 (Russian); translation in Ukrainian Math. J. 44 (1992), no. 10, 1292-1298. MR 94c:30025

[R3] Ryazanov V.I., Convergence of characteristics of quasiconformal mappings, Ukrain. Mat. Zh. 38 (1986), 200-204, 269 (Russian). MR 87h:30046

[S] SAKs S., Theory of the integral, New York, Dover Publ. Inc., 1964. MR 29:4850

[Sh] Shabat B.V., On generalized solutions of a PDE system, Mat. Sb. 17(59) (1945), 193-209 (Russian).

[T] TeichmüLler O., Untersuchungen über konforme and quasikonforme Abbildungen, Deutsche Math. 3 (1938), 621-678.

[W] Wiтtich H., Zum Beweis eines Satzes über quasikonforme Abbildungen, Math. Z. 51 (1948), 275-288. MR 10:241e

Institute of Applied Mathematics and Mechanics, NAS of Ukraine, ul. Roze LuxemBURG 74, 340114, Donetsk, UKRAINE

Current address: Department of Mathematics, P.O. Box 4 (Yliopistonkatu 5), FIN-00014 University of Helsinki, Finland

E-mail address: ryaz@iamm.ac.donetsk.ua

E-mail address: ryazanov@www.math.helsinki.fi

Department of Mathematics, P.O. Box 4 (Yliopistonkatu 5), Fin-00014 University of Helsinki, Finland

E-mail address: vuorinen@csc.fi 